\title{
Theoretical Value of Hubble's constant is a Salient Feature of Experimental Results- New Insight in to Origin of Universe
}

\author{
Siva Prasad Kodukula \\ ORCID-ID 0000-0003-3589-2216 \\ Visakhapatnam,India \\ sivkod@gmail.com
}

\begin{abstract}
Specifically this work is based on the concept of 'dark energy' as a phenomenal effect of expansion through which a theoretical value of Hubble's constant i.e. $\mathrm{H}_{\mathrm{th}}$ has been introduced. It is a constant that is based on a postulate that the square root of ratio of the Siva's constant ' $\mathrm{K}$ ' and Hubble's constant ' $\mathrm{H}$ ' is exactly equal to the diameter of the neutral hydrogen atom. That is the, exact theoretical value of Hubble's constant. This $\mathrm{H}_{\text {th }}$ is always constant .However, space-time conversions and the changes in the velocity of light will affect the distance and velocity in Hubble's equation. Deviation factors in velocity, distance and the Hubble's constant have been calculated separately which are affected by change of velocity of light with expansion of space time. The observations are related to red shift. So the change factor in terms of Hubble's constant has been calculated with respect to red shift. Thus a factor which affects the theoretical Hubble's constant has been calculated to find the exact value of Hubble's constant that satisfies the experimental results. The present theoretical value of Hubble's constant has been calculated as $\mathrm{H}=69.6 \mathrm{Km} \cdot \mathrm{s}^{-1} \mathrm{Mpc}^{-1}$

The experimental results by plank, ACT (Atacama Cosmology Telescope), WMAP (Wilkinson Microwave Anisotropy Probe) etc. experiments are at par with the theoretical result. Thus the ontology of space time introduced in this theoretical work supposed to be correct and leads to a new theory of origin of universe for which a brief description has been provided.
\end{abstract}

Key words: Hubble's constant ' $H$ ', space time, velocity of light, parsec, expanding universe, Siva's constant ' $\mathrm{K}$ '.

\section{Introduction}

Hubble's parameter is an important aspect of cosmology. It is introduced in the year 1920[1].From 2001 to till date if we take the experimental value of Hubble's constant It was varying from 60 to $70 \mathrm{~km} \cdot \mathrm{sec}^{-1} \cdot \mathrm{Mpc}^{-1}$ by various methods like plank collaboration data, $\Lambda \mathrm{CDM}$ (Lambda-Cold Dark Matter),ACT(Atacama Cosmology Telescope) and WMAP (Wilkinson Microwave Anisotropy Probe)etc. Their best Estimate is $69.6 \pm 0.7 \mathrm{~km} \cdot \mathrm{s}^{-1} \cdot \mathrm{Mpc}^{-1}$ [2]. ACT data with large scale information WMAP, got a value of $67.6 \pm 1.1 \mathrm{~km} \cdot \mathrm{s}^{-1} \cdot \mathrm{Mpc}^{-1}$ and ACT alone gives $67.9 \pm 1.5 \mathrm{~km} \cdot \mathrm{s}^{-1} \cdot \mathrm{Mpc}^{-1}[3]$ 
.Experimental physicists are trying to get more precision measurements by rectifying errors. But there is no theoretical frame work for Hubble's constant. In this paper an attempt has been made for getting a theoretical value of Hubble's constant which can be considered exact targeted value of the experiment. It is considered that the variation in Hubble's parameter is due to a phenomenon by which 'time' converts in to 'space'. Observations shows it as an effect. Due to this effect a constant value known as theoretical value of Hubble's constant i.e. $\mathrm{H}_{\mathrm{th}}$ is changing with a factor. A new concept of 'dark energy'[4] concluded that cosmological catastrophe regarding expansion of universe is due to conversion of space in to time and 'space time' converts in to 'mass'. It is against to the notion of a concept that space expands with time. Thus its calculations showed that mass energy density of the universe is constant and observed as the matter is flying apart in space. It can be verified experimentally by observing light velocity with the expansion of the universe. Also, it has derived an equation how the velocity of light changes with expansion of the universe. It is basically dependent on three postulates. The first one is 'space' and 'time' are two separate fundamental entities equated by equation $t^{3}=3 d^{2}$. Second one is space time is a separate form of energy with a combination of space and time which are interchangeable as one decreases, the other increases. Third one is space time is existed in such a way which holds the Hubble's law interpreted by equation $\mathrm{V}=\mathrm{Hd}$. By one more postulate, the Hubble's constant and Siva's constant ' $\mathrm{K}$ ' are related by size of hydrogen atom [4]. This relation concluded a theoretical value of Hubble's constant. With these four postulates, Cosmic Micro wave Back ground Radiation (CMBR) has been calculated[4]. It is at par with the value of CMBR value that was verified experimentally and Got Nobel prize [5].

So the theoretical value of ' $\mathrm{H}$ ' i.e. $\mathrm{H}_{\mathrm{th}}$ with the above postulates is correct.

Now in this paper, let us make theoretical value of ' $\mathrm{H}$ ' termed as $\mathrm{H}_{\mathrm{th}}$ compatible to experimental efforts.

"It is to be noted that all the previous work [4] related to this paper emphasizes that space time is different and space is different. Volume of 'space time' and volume of 'space' concerned to that 'space time' are considered as same. This will not obey the mathematics of volume of fourdimensional universe which is different from volume of space concerned to that space time. In space time, if space increases, time decreases but in space time, if volume increases, the space time also will increase and satisfy $t^{3}=3 d^{2}$. Space time increases in the case of merging of two are more similar space times. At the same time if the phenomenon is applied to one specific space time which cannot be increased are decreased, the space and time will interchange since the time is having a natural property to flow. Further papers will elaborate this phenomenon to synchronize General Relativity with Quantum mechanics. 
In one perspective, it supports 'big bang'. It differs with the 'big bang' in the aspect of 'bang' .But it supports the idea that universe started with a single point with a diameter equal to Planck length. Its calculation for CMBR gives a value of $2.68^{\circ} \mathrm{K}$ [4] and in another perspective the matter is being created continuously and compensates expansion to keep the mass energy density constant. In this aspect it supports Steady state, but differs in the aspect that Universe started with a single Planck mass. Its roots are linked with a concept that synchronizes general Relativity and Quantum mechanics. Now it is not the point of discussion. Further papers will elaborate it.

\section{Experimental value of ' $H$ ' from theoretical value of Hubble's constant i.e. ' $H_{\text {th }}$ '}

As per the paper[4], It is postulated that the exact value of Hubble's constant must be equal to a theoretical value which shows relation between Siva's constant ' $K$ ' and diameter of neutral Hydrogen atom.

Thus mathematically it concluded an equation[4],

$$
\mathrm{H}_{\mathrm{th}}=\frac{\mathrm{K}}{\mathrm{d}^{2}}=4.1394908592 \times 10^{-19} \mathrm{sec}^{-1}
$$

As per the paper [4], we have an equation,

$$
\mathrm{V}=3 \mathrm{Hc}_{\mathrm{O}}^{3}
$$

Where -

' $\mathrm{V}$ ' is velocity of galaxy which is receding away from us.

' $\mathrm{H}$ ' is Hubble's constant must be theoretical value of Hubble's constant i.e. ' $\mathrm{H}_{\mathrm{th}}$ '

' $\mathrm{C}_{\mathrm{o}}$ ' is velocity of light received from that receding galaxy.

It elaborates that velocity of light changes with the expanding universe.

Here, we should note that these two conclusions are arrived by assuming that space and time are treated as space time continuum but the space will increase with decrease in time and the theoretical value(exact value) of Hubble's constant is the ratio of Siva's constant ' $\mathrm{K}$ ' and the square of diameter of neutral hydrogen atom[4].

These assumptions are right if the theoretical value of Hubble's constant in terms of experimental value of Hubble's constant.

Already, the value of CMBR Calculated by this ' $\mathrm{H}_{\mathrm{th}}$ ' value is matching with the experimental value of CMBR [5]. It is one of the proofs that support the above assumptions. 
Now let us interpret the ' $\mathrm{H}_{\mathrm{th}}{ }^{\prime}$ in terms of the value which can be detected by observation:

We know that the expanding universe is described by Hubble's law V $=\mathrm{Hd}$

Here ' $\mathrm{V}$ ' is velocity of receding galaxy existed at a distance' $\mathrm{d}$ ' and the ' $\mathrm{H}$ ' is proportionality constant.

The experiment to find the velocity is done by observing the light from that receding galaxy at a particular moment when it is at distance'd'. The receding galaxy from the observer shows a 'red shift' in the spectrum due to 'Doppler Effect'. When Doppler Effect is applied to expanding universe, the basic formula used is $z c=v$, and $\mathrm{z}$ changes with distance. Thus by red shift we can find ' $\mathrm{V}$ ' is proportional to ' $\mathrm{d}$ ' and the proportionality constant is ' $\mathrm{H}$ ' called as Hubble's constant. In this observation, ' $c$ ' is constant and ' $z$ ' is red shift. Means, these observations have to be corrected if velocity of light changes.

As per (2), light velocity is changing with distance since $\frac{\mathrm{V}}{\mathrm{H}}=\mathrm{d}$

So the Mega parsec (Mpc) value also will also change accordingly.

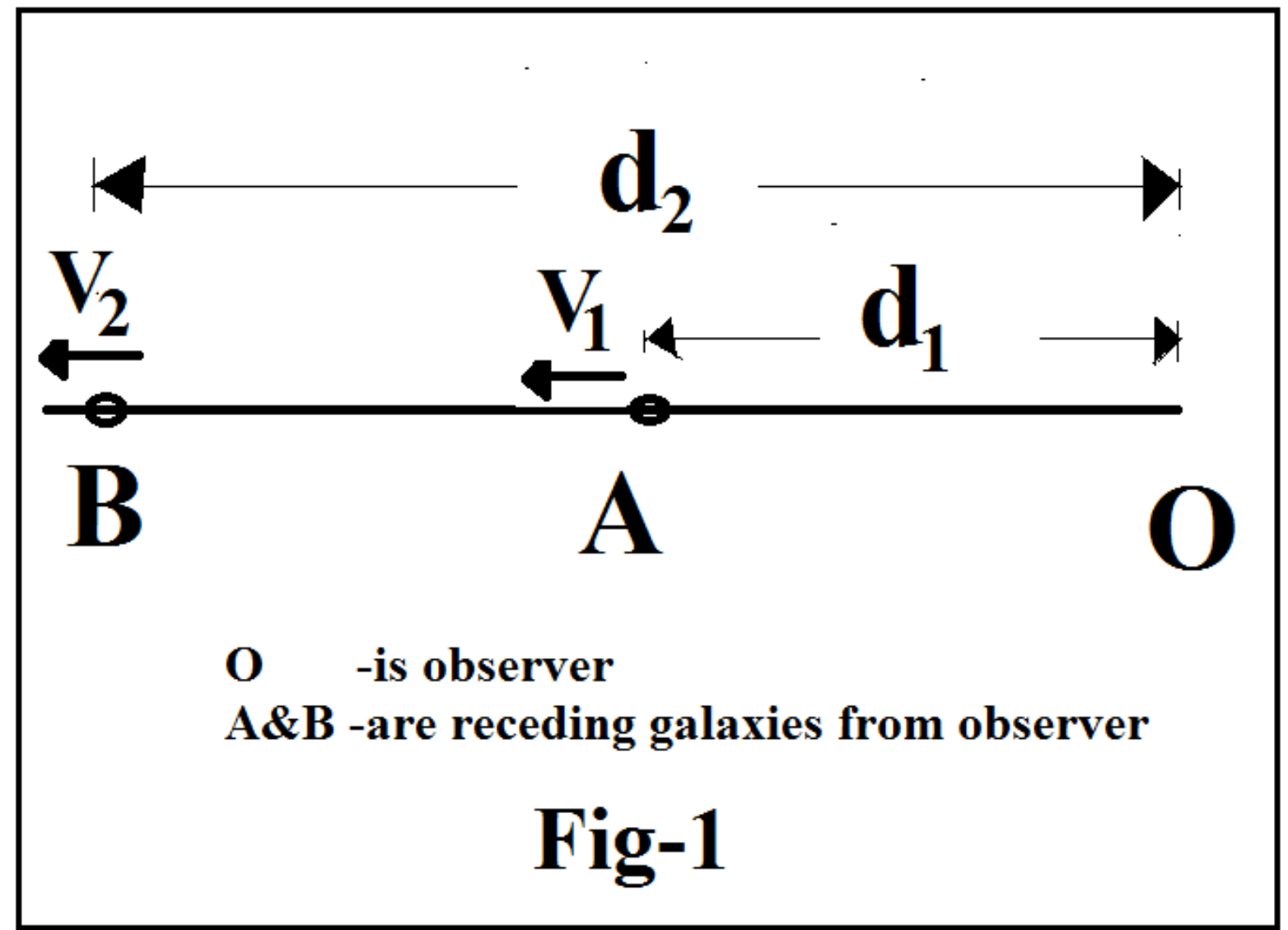


Fig.1 Shows the that galaxy ' $A$ ' at a distance 'd1' from observer 'o' will have a velocity ' $V_{1}$ ' and can be calculated by its red shift ' $\mathrm{z}$ ' by the equation $\mathrm{zc}=\mathrm{v}$.Here the velocity of light is constant even in the expansion also. Means ' $c$ ' will shows the linear relation between space and time. As' $d$ ' increases, time also will increase.

But as per space time equivalence principle, the distance and time are not equal. So linearity will not exist.. As per that, the relation between distance and time is

$$
\mathrm{t}^{3}=3 \mathrm{~d}^{2}
$$

If it is linear, light velocity is constant. If it is not liner, velocity of light will be changed.

Now let us suppose that the galaxy has moved from 'A' to 'B' located at a distance $d_{2}$ from observer ' $\mathrm{O}$ '. Now the velocity will become $\mathrm{V}_{2}$.

$$
\begin{gathered}
\mathrm{V}_{1}=\mathrm{Hd}_{1} \\
\mathrm{~V}_{2}=\mathrm{Hd}_{2} \\
\mathrm{~V}_{2}-\mathrm{V}_{1}=\mathrm{H}\left(\mathrm{d}_{2}-\mathrm{d}_{1}\right)
\end{gathered}
$$

Say $\Delta \mathrm{V}$ is the factor by which velocity $\mathrm{V}_{1}$ increased and $\Delta \mathrm{d}$ is the factor by which distance $\mathrm{d}_{1}$ increased

$$
\therefore \Delta \mathrm{V}=\frac{\mathrm{V}_{2}-\mathrm{V}_{1}}{\mathrm{~V}_{1}}
$$

Similarly,

$$
\Delta \mathrm{d}=\frac{\mathrm{d}_{2}-\mathrm{d}_{1}}{\mathrm{~d}_{1}}
$$

So finally Hubble's law can be re written as

$$
\mathrm{V}_{1} \Delta \mathrm{V}=\mathrm{Hd}_{1} \Delta \mathrm{d}
$$

But $\Delta \mathrm{d}$ effects $\mathrm{H}$ also. Say the change factor in $\mathrm{H}$ as $\Delta \mathrm{H}$

In terms of Hubble's equation. 


$$
\Delta V=\Delta H \Delta d
$$

In equation(10) Velocity is always Observed and supported by Doppler red shift according to the equation $\mathrm{zc}=\mathrm{V}$ where ' $\mathrm{c}$ ' is constant. Right side of the equation is actual and follows First postulate that $\mathrm{d}$ and $\mathrm{t}$ exchanges as per equation(3). But it will not satisfy Hubble's law and equation $\mathrm{V}=\mathrm{Hd}$ ' In order to satisfy $\mathrm{V}=\mathrm{Hd}$, In equation (10) right side term must be $\frac{\Delta \mathrm{d}}{\Delta \mathrm{H}}$ Instead of $\Delta H \Delta d$ Thus we can rewrite equation (10) as-

$$
\frac{\Delta \mathrm{d}}{\Delta \mathrm{H}}=\Delta \mathrm{V}
$$

It follows second postulate that says space and time are directly proportional ('space time' is increasing . space and time are not exchanging) and follows the equation (3) i.e. $\mathrm{t}^{3}=3 \mathrm{~d}^{2}$

Now the Hubble's equation $\mathrm{V}=\mathrm{Hd}$ is valid with red shift.

If we elaborate equation (11),

$$
\Delta \mathrm{V} . \mathrm{V}=\frac{\mathrm{H}}{\Delta \mathrm{H}} \times \mathrm{d} \times \Delta \mathrm{d}
$$

Now let us calculate $\Delta \mathrm{d} \Delta \mathrm{H}$ and $\Delta \mathrm{V}$

Let us say $\Delta \mathrm{V}$ is the factor by which distance ' $\mathrm{d}$ ' has been increased

But it is not reality. As per law of conservation of energy space time can not be created since it is also a form of energy. So its space and time and exchange mutually as explained in the first postulate this will create change in distance and velocity of light. Here not only space and time but also mass also exchanges as explained in paper[4].Mass is formed due to contraction of space time. So finally time converts in to space and 'space time' converts in to mass and the mass energy density will be constant. This will result in to a phenomenon[4] that keeps space time constant and exchange of time in to space so that equation (3), (12) and Hubble's law i.e $\mathrm{V}=\mathrm{Hd}$ are valid. That will be observed as expansion by red shift.

In order to obey equation (12) this change will be evident in the change of $\Delta \mathrm{d} \Delta \mathrm{H}$ and $\Delta \mathrm{V}$

i)This change can be calculated for a distance of 1 Mega parsec.

As per space time equivalence principle [4]

We have equation (3) 


$$
\begin{gathered}
\mathrm{t}^{3}=3 \mathrm{~d}^{2} \\
\therefore \mathrm{t}=3^{\frac{1}{3}} \mathrm{~d}^{\frac{2}{3}}
\end{gathered}
$$

Let us calculate it for 1Mega parsec [6]

$$
\begin{gathered}
\therefore \mathrm{d}=1 \mathrm{Mpc}=3.085677581 \times 10^{22} \mathrm{~m} \\
\therefore \mathrm{t}=3^{\frac{1}{3}}\left(3.085677581 \times 10^{22}\right)^{\frac{2}{3}} \\
\therefore \mathrm{t}=1.4422495703 \times 9.8378537819 \times 10^{14} \\
\therefore \mathrm{t}=1.4422495703 \times 9.8378537819 \times 10^{14} \\
\therefore \mathrm{t}=1.4188640390 \times 10^{15} \mathrm{sec}
\end{gathered}
$$

We know $1 \mathrm{Mpc}$ is the celestial distance covered by the light with velocity $2.99792458 \times 10^{8} \mathrm{mt} \cdot \mathrm{sec}^{-1}$ and distance $3.085677581 \times 10^{22} \mathrm{mts}[6]$

Let us find the velocity of light covered the distance $3.085677581 \times 10^{22} \mathrm{mts}$ in $1.4188640390 \times 10^{15} \mathrm{sec}$.

$$
\therefore c=\frac{d}{t}=\frac{3.085677581 \times 10^{22}}{1.4188640390 \times 10^{15}}=2.1747521230 \times 10^{7}{\mathrm{~m} . \mathrm{sec}^{-1}}^{-1}
$$

In this time reduced if we think that time not reduced the same can be interpret as 'distance' increased.

Let us take for the same time, distance increased due to slow of light velocity.

The factor $\Delta \mathrm{d}$ in the distance $1 \mathrm{mpec}$ due to space time conversion $=$

$$
\begin{gathered}
\frac{2.99792458 \times 10^{8}}{0.21747521230 \times 10^{8}}=13.7851323298 \\
\therefore \Delta d=13.7851323298
\end{gathered}
$$

\section{ii) Leus find $\Delta H$}

$\Delta \mathrm{H}$ is the factor by which ' $\mathrm{H}$ ' has been reduced

Increase in $\Delta \mathrm{d}$ is due to the reduction in time. ' $\mathrm{H}$ ' is nothing but a function of time. 
In equation $\quad \mathrm{V}=\mathrm{Hd}$, ' $\mathrm{d}$ 'is parsec. it is decreasing by 13.78 times and ' $\mathrm{H}$ ' is a function of time. Parsec is also a function of time. its value decreased due to increase in time. Increase in time reduces value of $\mathrm{H}$. Let us say the change factor as $\Delta \mathrm{H}$

We have equation (17)

$$
\Delta \mathrm{d}=13.7851323298
$$

We know

$$
\Delta \mathrm{H}=\frac{1}{\Delta \mathrm{t}}
$$

And as per (3)

$$
\mathrm{t}^{3}=3 \mathrm{~d}^{2}
$$

Convert $\Delta \mathrm{t}$ in to $\Delta \mathrm{d}$

$$
\therefore \Delta \mathrm{H}=\sqrt[3]{\frac{\mathrm{d}}{3}}
$$

Substitute $d=13.7851323298 m t$.

$$
\begin{array}{r}
\Delta H=\sqrt[3]{\frac{13.7851323298}{3}} \\
\Delta H=1.6625060264
\end{array}
$$

\section{iii) Letus calculate $\Delta \mathrm{V}$}

if there is no change in ' $\mathrm{d}$ ' i.e $\Delta \mathrm{d}=1$ then so $\Delta H=1$

Velocity ' $\mathrm{V}$ ' should not change but even then velocity changes because $\Delta d=1$

$$
\begin{gathered}
\therefore \Delta \mathrm{V}=\sqrt[3]{\frac{1}{3}} \\
\therefore \Delta \mathrm{V}=0.69336127435
\end{gathered}
$$

Thus we can find the value $\mathrm{s}$

$$
\begin{gathered}
\Delta d=13.7851323298 \\
\Delta H=1.6625060264 \\
\Delta V=0.69336127435
\end{gathered}
$$


We have equation(12)

$$
\mathrm{V} \times \Delta \mathrm{V}=\left(\frac{\mathrm{H}}{\Delta \mathrm{H}}\right) \times(\mathrm{d} \times \Delta \mathrm{d})
$$

The same equation can be written as

$$
\mathrm{V}=\left(\frac{\Delta \mathrm{d}}{\Delta \mathrm{V} \times \Delta \mathrm{H}}\right) \mathrm{H}_{\mathrm{th}} \times \mathrm{d}
$$

Thus as a whole $\mathrm{H}_{\text {th }}$ will be affected by factor $\frac{\Delta \mathrm{d}}{\Delta \mathrm{V} \times \Delta \mathrm{H}}$

$$
\frac{\Delta \mathrm{d}}{\Delta \mathrm{V} \times \Delta \mathrm{H}}=5.4489367478
$$

Thus for $1 \mathrm{Mpc}$ distance, the factor is $\mathbf{5 . 4 5}$

In these calculations we have considered light velocity is constant. So $\Delta \mathrm{d}$ changes and concerned ' $H$ ' and $\Delta V$ also changes. Since light velocity is constant the value of one megaparsec is correct and the factor is 5.45. So ' $\mathrm{H}$ ' value in $\mathrm{V}=\mathrm{Hd}$ can bcalculated

Here velocity is ' $\mathrm{V}$ '

The total change in factor ' $\mathrm{H}$ ' as per (21) is $\frac{\Delta \mathrm{d}}{\Delta \mathrm{V} \times \Delta \mathrm{H}}=5.4489367478$

Now with this we can find the ' $\mathrm{H}$ ' by equation (20)

$$
\begin{gathered}
\mathrm{H}=\mathrm{H}_{\mathrm{th}} \times \frac{\Delta \mathrm{d}}{\Delta \mathrm{V} \times \Delta \mathrm{H}}=4.1394908592 \times 10^{-19} \times 5.4489367478 \\
\therefore \mathrm{H}=\mathrm{H}_{\mathrm{th}} \times \frac{\Delta \mathrm{d}}{\Delta \mathrm{V} \times \Delta \mathrm{H}}=2.2555823860 \times 10^{-18} \mathrm{sec}^{-1}
\end{gathered}
$$

We have value of 1 parsec $=3.085677581491 \times 10^{16} \mathrm{~m}$

$$
\begin{gathered}
\therefore \mathrm{H}=2.2555823860 \times 10^{-18} \times 3.085677581491 \times 10^{16}{\mathrm{~m} . \mathrm{s}^{-1} \mathrm{pc}^{-1}}^{\therefore \mathrm{H}=69.6 \mathrm{Km}_{\mathrm{s}}{ }^{-1} \mathrm{Mpc}^{-1}}
\end{gathered}
$$

In other words,

We have the equation $\quad \mathrm{V}=\mathrm{Hd}$

Increase in $\Delta \mathrm{d}$ should decrease in ' $\mathrm{H}$ ' to keep $\mathrm{V}=\mathrm{Hd}$ 


$$
\therefore \mathrm{V}=\frac{\mathrm{H}}{\Delta \mathrm{H} \cdot \Delta \mathrm{d}}
$$

But still $\mathrm{V}=\mathrm{Hd}$ will not be satisfied because in above equation $\Delta \mathrm{d}=13.7851323298$ In that place if we keep $\Delta \mathrm{d}=1, \mathrm{~V}=\mathrm{Hd}$ must be valid. But it will not be valid since if $\Delta \mathrm{d}=1$ $\Delta \mathrm{V}=0.69336127435$ so to validate $\mathrm{V}=\mathrm{Hd}$ ' $\mathrm{H}$ ' should be multiplied by 0.69 .

So totally the $\mathrm{V}=\mathrm{Hd}$ equation will be affected by these factors

The equation $\mathrm{V}=\mathrm{Hd}$ can be elaborated as

$$
\mathrm{V}=0.6933612744 \times \frac{\mathrm{H}}{\Delta \mathrm{H}} \times \mathrm{d} . \Delta .
$$

As $\Delta \mathrm{d}$ increases or decreases the $\Delta \mathrm{H}$ explained by equation will change and $\mathrm{H}$ will be affected by the total factor .

$$
\frac{\Delta \mathrm{d}}{\Delta \mathrm{H}} \times 0.69
$$

\section{Discussion}

Then what is the exact value ? Can we theoretically predict the exact value of Hubble's constant? In the paper[4] we have observed the ratio of Siva's constant and Hubble's constant is a value close to diameter of hydrogen atom. At that time we do not have any supporting logic to accept it .So we have postulated the value is exact and concluded that ' $\mathrm{H}$ ' as exact or theoretical value. With this, value we have calculated $H_{\text {th }}$ and CMBR values. The CMBR is almost equal to experimentally verified value of CMBR[5]. Thus the postulate supported the logical idea to lead further research.

In this paper the same postulates have been considered they are

1. The first one is space and time are two separate fundamental entities equated by equation(3) i.e. $\mathrm{t}^{3}=3 \mathrm{~d}^{2}$.

2. Second one is space time is a separate form of energy with a combination of space and time which are interchangeable as one decreases ,the other increases.

3. Third one is space time is existed in such a way which holds the Hobbles law interpreted by equation $\mathrm{V}=\mathrm{Hd}$

4. One more postulate, the Hubble's constant ' $\mathrm{H}$ ' and Siva's constant ' $\mathrm{K}$ ' are related by diameter of hydrogen atom. This relation concluded a theoretical value of Hubble's constant $\mathrm{H}_{\text {th }}$. 
And Concluded that space time exist with $\quad \mathrm{V}=\mathrm{Hd}$

As per the equation (26), Value of ' $\mathrm{H}$ ' will change with a factor $\frac{\Delta d}{\Delta H} \times 0.69$

It is due to the consequence of phenomena by which space time converts in to one another and the mass energy density remains constant explained as an alternative to dark energy [4].

The change in ' $\mathrm{H}$ ' due to the calculated factor gives the exact or theoretical value of ' $\mathrm{H}$ ' at a specific moment of red shift observation. As the red shift changes, the velocity will change since $z \mathrm{c}=\mathrm{v}$ where ' $c$ ' is constant. For this value of red shift, there exist a factor affecting ' $H$ ' in Hubble's equation and that value will match with the observed red shift. Thus we can find the exact value of ' $\mathrm{H}$ ' and concerned velocity ' $\mathrm{V}$ ' and distance ' $\mathrm{d}$ '. This value of ' $\mathrm{H}$ ' is with in the range of experimental results[2][3]. So this must be considered as exact value supported by theory and the experimental results also support the postulates. This must be a theoretical back ground for Hubble's constant and its variation with velocity. This may be helpful for experimental scientists on this field to find any errors and to eliminate them in calculations of these experiments.

\section{Origin of Universe and Hubble's parameter}

Hubble's parameter is the most important aspect of origin of universe theories. If we go through the sequential order of the evolution of these ideas and concepts on origin of universe, we can understand where we are and the direction of our research. I considered the order of these historical discussions on origin of the universe for introduction as per the paper [7]

"In the 1920s Edwin P. Hubble measured the recession velocities of 18 spiral galaxies with a reasonably well-known distance, and found that all the velocities increased nearly with distance, $\mathrm{V}=\mathrm{H}_{0} \mathrm{~d}$, This is Hubble's law, and $\mathrm{H}_{0}$ is called the Hubble parameter. Einstein published his General Theory of Relativity in 1917, but the only solution he found to the highly nonlinear differential equations was static. Immediately after General Relativity became known, Willem de Sitter (1872-1934) found and published an exponentially expanding solution to Einstein's equations for the special case of empty space time. In 1922 Alexandr Friedman (18881925) found a range of solutions, intermediate to Einstein's static solution and de Sitter's solution.

Friedman's solutions did not gain general recognition until after his death when they were confirmed by an independent derivation (in 1927) by Georges Lemaitre (1894-1966). in 1934 did Robertson and Walker construct the RW metric to match the general geometrical structure of the Einstein's tensor $G_{\mu \nu}$. Today the standard model of cosmology is based on the Friedman Lemaitre equations (FL) and the RW metric. In the static universe of Einstein a(t) is constant and the age of the Universe is infinite. The cosmological constant $\Lambda$ corresponds to a tiny correction 
to the geometry of the Universe which adds enough repulsion to make the Universe static so he corrected it as $\Lambda g_{\mu \nu}$ to make it Lorenz invariant. Unfortunately Einstein was wrong, Hubble showed that the universe was expanding, and Einstein admitted $\Lambda$ had been a blunder. The idea of expanding universe supported the idea that the universe started from a dense radiation dominant point immediately after a singularity point. The Cosmic Microwave Background Radiation (CMBR) which has existed since the era of radiation domination could be predicted, and was indeed predicted in 1948 at almost the right temperature by George Gamow (1904-1968), Ralph Alpher (1921-2007) and Robert Herman (1914-1997).Finally The temperature of this radiation was calculated and verified by experiments as $\mathrm{T}_{0}=2.725^{\circ} \mathrm{K}$ with precision"[5].

Hubble's work [1] on expanding universe is the basis for origination of continuous creation of matter concept. In 1948 paper Bondi explained that the average rate of creation is determined by the rate of expansion and the density of the universe .is. Approximately 10-43g.persec.percm3.[8] This calculation is based on Hubble's mass distribution calculation[1] .It was mathematically substantiated by F. Hoyle [9]by using the concept of continuous creation of matter, with in the frame work of general relativity, but without introducing a cosmological constant, a universe satisfying a cosmological principle that shows the required expansion prosperities and in which the localized condensations are continuously formed. The ideas originally proposed to discuss continuous creation of matter are reconsidered in the context of big bang cosmological models [10]. It is shown that the singularity-free big bang models are possible under the modified field equations of General relativity but the case is made up of that the matter creation takes place in several mini bangs at different epochs rather than in one big bang.[10]Later Hoyle Narlikar theory was proposed with varying Gravitational constant ' $G$ '. They claimed an equation for CMBR $3 \mathrm{~K}$ radiation with this varying ' $G$ ' ratio. But They them self concluded that the difference between ' $G$ constant' and G-varying cosmologies are in general of two kinds: geometrical and physical. In principle, it should be possible to test these differences to see which cosmology is right. In practice, however, the situation is more complicated because these differences get mixed up[11].How ever big bang was boosted up by Nobel winning experimental result of cosmic micro wave back ground radiation [5]Some papers explain that superluminal velocities in expanding universe within the frame work of Special relativity [12]. But velocities more than that of light are nothing to do with this explanations. It is classical only. I have not touched that part since the analysis started from a classical approach nowhere applied the special relativity for velocities.

Now in the paper [4] it is explained that universe is existed with a diameter equal to planck length. The mass energy density of the universe is constant at any stage of the universe. It is explained that mass is a form of space time. So, continuous creation of mass affect the space time. At the same time space and time are interchangeable. Thus the calculations shown in the paper [4] described cosmological catastrophe as a phenomenal effect of expansion. The cosmological model that describe 
the origin of universe based on this phenomenal effect is different from big bang, steady state and other theories explained above. It supports big bang when space time is with diameter at Planck diameter and due to conversion of time in to space and to maintain density of mass energy constant it shown like expansion for observations. This effect can be concluded by change in Hubble's constant with a factor which is at par with the experimental results[2][3]. So this result it self is sufficient to form a new model for origin of universe. Further papers will elaborate this model from the fundamentals of quantum mechanics and General relativity by synchronising them.

\section{Conclusions}

1. In Hubble's expanding universe formula $\mathrm{V}=\mathrm{Hd}$, ' $\mathrm{V}$ ' is velocity of the receding galaxy and ' $d$ ' is the distance of that galaxy from observer. It says that ' $V$ ' is proportional to ' $d$ ' and ' $H$ ' is constant of proportionality. In this paper it is concluded that there is a theoretical value of ' $\mathrm{H}$ ' called as $\mathrm{H}_{\text {th }}$ and it will vary with a factor $\frac{\Delta \mathrm{d}}{\Delta \mathrm{V} \times \Delta \mathrm{H}}$

Thus the equation can be written as- $\mathrm{V}=\left(\frac{\Delta \mathrm{d}}{\Delta \mathrm{V} \times \Delta \mathrm{H}}\right) \mathrm{H}_{\mathrm{th}} \times \mathrm{d}$

$\Delta \mathrm{d}, \Delta \mathrm{H}$ and $\Delta \mathrm{V} \quad$ are factors by which ' $\mathrm{d}$ ', ' $\mathrm{H}$ ' and ' $\mathrm{V}$ '. These factors will vary with the distance from the observer. The above integrated factor changes with the red shift. We can find the exact value of Hubble's constant by red shift. It says that value in Mega parsecs depends on light velocity which changes with the distance. For present value of $1 \mathrm{Mpc}$, the Hubble's constant has been calculated as-

$\mathrm{H}=69.6 \mathrm{Km}^{-1} \mathrm{Mpc}^{-1}$ (or $) 2.2555823860 \times 10^{-18} \mathrm{sec}^{-1}$.This is exact value of Hubble's constant.

2. Experimental values are supporting this theoretical frame work for calculating exact value of ' $\mathrm{H}$ '. So undoubtedly, the postulates, concepts and the new concept of space time in this theoretical frame work are correct. This may turn physics to a new dimension.

3. This concept of space time is a small deviation in space time ontology of general relativity. This small deviation and the concerned calculations forms quantum particles with space time by which grand unification can be explained. Not only that, this basic change in space time concept will explain how the space time will be enlarged by merging of two or more fundamental particles made up of indivisible space times. Further, it will explain that how these quantum space times will form a smooth space time explained by general relativity. Thus it gives a solution for synchronisation of quantum mechanics with general relativity. Further works will elaborate these concepts for advancement of modern physics. 


\section{References}

1. E. Hubble, The realm of the Nebulae, (Oxford,1936),p-189

2. C. L. Bennett," The One percent concordance Hubble Constant", The Astrophysical Journal, 794:135 (8pp), 2014 October 20 https://iopscience.iop.org/article/10.1088/0004-637X/794/2/135/pdf

3. Steve K. Choil, "The Atcama Cosmology Telescope: A Measurement of the Cosmic Microwave Background Power spectra at 98and 150 Ghz". Arxiv Preprint July 14, 2020, https://arxiv.org/abs/2007.07289v1

4. S.P.Kodukula, Dark Energy is a Phenomenal Effect of the Expanding Universe-Possibility for Experimental Verification, Research square Preprint: https://www.researchsquare.com/article/rs-40479/v2

5. A. A. Penzias,R. W. Wilson, A measurement of Excess Antenna Temperature at 4080 $\mathrm{Mc} / \mathrm{s}$, ,APJ letters to the editor,vol.142,no.1(1965),p/419-320 http://articles.adsabs.harvard.edu/pdf/1965ApJ...142..419P

6. Andrej Prsa et.al., "Nominal values for selected solar and planetary quantities: IAU 2015Resolution B3", arXiv:1605.09788v1 [astro-ph.SR], 31 May2016. http://www.iau.org/static/resolutions/IAU2015_English.pdf.

7. Matts Roos, "Expansion of the Universe - Standard Big Bang Model”, arXiv:0802.2005v1 [astro-ph] 14 Feb 2008, https://arxiv.org/pdf/0802.2005.pdf

8. H.Bondi \& T.Gold "The steady-state theory of the Expanding Universe", MNRAS,3 (1948) https://academic.oup.com/mnras/article/108/3/252/2603423

9. F.Hoyle," A new model for the Expanding Universe",MNRAS,5(1948) https://academic.oup.com/mnras/article/108/5/372/2601825

10. J.V.Narlikar,"Minibangs in cosmology and astrophysics",Pramana,viol2.no.3(1974),pp158170, https://doi.org/10.1007/BF02847326

11. V.M. Canuto \& J.V.Narlikar, Cosmological Tests of the Hoyle-Narlikar conformal Gravity,APJ,236:6-23.(1980), https://pubs.giss.nasa.gov/docs/1980/1980_Canuto_ca03600a.pdf

12. Tamara M. Davis and Charles H. Line weaver, Expanding Confusion: Common Misconceptions of Cosmological Horizons and the Superluminal Expansion of the Universe, Publications of the Astronomical Society of Australia, 2004, 21, 97-109,2003, www.publish.csiro.au/journals/pasa, 